\title{
Dehydration as an Etiologic Factor of Halitosis: A Case-Control Study
}

\author{
Soo-Min $\mathrm{Ok}^{1}$, Sung-Hee Jeong ${ }^{1}$, Chang-Hyung Lee ${ }^{2}$ \\ 1Department of Oral Medicine, Dental Research Institute, Pusan National University Dental Hospital, Dental and Life Science \\ Institute, Pusan National University, School of Dentistry, Yangsan, Korea \\ ${ }^{2}$ Department of Rehabilitation Medicine, Research Institute for the Convergence of Biomedical Science and Technology, Pusan \\ National University Yangsan Hospital, Pusan National University School of Medicine, Yangsan, Korea
}

Received November 19, 2021

Revised December 3, 2021

Accepted December 3, 2021
Purpose: Salivation is considered to be an important factor in the control of halitosis, and the amount of salivation has been shown to be closely related to the level of hydration. The purpose of our study was to evaluate the relationship between dehydration and halitosis.

Methods: Twenty healthy young females with no dental problems were recruited. All participants were induced to become dehydrated and then over-hydrated. After inducing each hydration state, the severity of hydration and halitosis factor (organoleptic scores, amounts of resting and functional saliva, gas examinations, and tongue coatings) were measured. Hydration statuses were graded as dehydration, normal, or over-hydration according to urine osmolality. This was a cross sectional study with a cross over design.

Results: The dehydrated status was associated with higher organoleptic scores than the normal or over-hydrated status $(1.75 \pm 0.75$ vs. $0.87 \pm 0.63$, and $0.65 \pm 0.53$, respectively. $\mathrm{p}<0.05)$. Mean values of $\mathrm{CH}_{3} \mathrm{SH},\left(\mathrm{CH}_{3}\right)_{2} \mathrm{~S}$ in portable gas chromatography for the dehydrated, normal, and over-hydrated status were $11.70 \pm 37.00,6.75 \pm 13.50$, and $2.80 \pm 5.87 \mathrm{nmol} / \mathrm{mol}$, $10.50 \pm 15.59,7.25 \pm 10.87$, and $1.50 \pm 2.55 \mathrm{nmol} / \mathrm{mol}$, respectively. $\mathrm{p}>0.05) .\left(\mathrm{CH}_{3}\right)_{2} \mathrm{~S}(\mathrm{r}=0.410$, $\mathrm{p}=0.009$ ) showed a moderate positive correlation with dehydration status. The resting salivation rates were relatively lower for the dehydrated status than for the normal or overhydrated status ( $>0.05)$, and tongue coating results were also higher for the dehydrated status ( $\mathrm{p}>0.05)$.

Conclusions: Dehydration status appears to be positively correlated with a low resting salivation rate and high portable gas chromatography results. This shows that dehydration might be an etiologic factor of halitosis.

Key Words: Dehydration, Halitosis, Life style, Oral hygiene, Oral medicine, Saliva

\section{INTRODUCTION}

Humans emit a variety of volatile and non-volatile molecules in body fluids that are influenced by genetics, diet, stress, and disease [1]. Halitosis is a general term used to describe an unpleasant or offensive odor emanating from the oral cavity [2].

A complaint of halitosis may be objective, where an unpleasant odor produced anywhere in the body is emitted from the mouth and/or nose and is detectable to others, or subjective, where the odor is not detectable to others, but the patient complains of its presence. Any patient who complains of halitosis, either objective or subjective, should be considered as a "halitosis patient" [3].

Females usually show greater self-perception and feel more uncomfortable than males with halitosis [4]. Additionally, as one gets older there are increasing factors that can cause halitosis. Therefore, we decided it would be

Copyright (c) 2021 Korean Academy of Orofacial Pain and Oral Medicine

(c) This is an open-access article distributed under the terms of the Creative Commons Attribution Non-Commercial License (http://creativecommons.org/licenses/by-nc/4.0/), which permits unrestricted non-commercial use, distribution, and reproduction in any medium, provided the original work is properly cited. 
desirable to recruit young, female participants for this study, in order to reduce the probability of including other factors that can cause halitosis besides our intended variable and the focus of this study, hydration status [5,6].

Usually, halitosis worsens with age as the bacterial number or concentration of dimethyl sulfide $\left(\left[\mathrm{CH}_{3}\right]_{2} \mathrm{~S}\right)$ increases [7]. The vast majority of causes of halitosis lie within the oral cavity and the oropharynx (tongue coating, gingivitis, periodontitis, and tonsillitis). The formation of malodorous volatile sulfur compounds (VSCs), including hydrogen sulfide $\left(\mathrm{H}_{2} \mathrm{~S}\right)$ and methyl mercaptan $\left(\mathrm{CH}_{3} \mathrm{SH}\right)$, by bacteria residing within the oral cavity, and especially in the coating of the tongue, plays a predominant role [8]. In 10\% to 15\% of patients, halitosis has an extra-oral cause; this is primarily due to the presence of $\left(\mathrm{CH}_{3}\right)_{2} \mathrm{~S}$ [7,9]. However, even in a young and healthy person, halitosis can be present without evidence of bacterial colonization or the formation of VSCs; this is called physiologic halitosis. In previous studies, the existence of physiologic halitosis was found to vary with such conditions as the time of day [10]. Therefore, it is considered likely that halitosis results from the summation of organic and physiologic factors.

The severity of halitosis is usually determined subjectively. Several methods have been proposed to measure the severity of halitosis objectively; these include measuring the concentrations of oral gases possibly responsible for halitosis. Portable gas chromatography such as OralChroma (Abilit Corp., Osaka, Japan) judges the severity of halitosis by measuring the concentrations of VSCs present in the expiratory gas [11].

Rates of resting and stimulated saliva flow can affect the severity of halitosis [1]. In addition, the severity of halitosis can be estimated by noting the amount of tongue coating, which contains germs and organic waste products [8]. In general, pathologic conditions causing halitosis have been localized to the mouth, ear, nose, throat, stomach, and lungs. The ingestion of certain medications and foods has also been implicated. Any condition that favors bacterial colonization can cause halitosis [12]. In addition, systemic illness might result in the formation of malodorous metabolic by-products. Saliva is believed to reduce the number of bacteria present in the oral cavity, and thus, the severity of halitosis. Saliva plays an important role in the oral cavity, lubricating tissue and facilitating deglutition and cleansing. Furthermore, some of the electrolytes and proteins present in saliva possess antibacterial properties [13-16].

Salivation is considered to be an important factor in the control of halitosis, and the amount of salivation has been shown to be closely related to the level of hydration [17]. Reduced antibacterial activity by saliva is thought to result in halitosis secondary to VSC production [18]. In addition, decreased cleansing by saliva might result in an increased amount of tongue coating.

We suspected that dehydration could be an important factor in the development of halitosis, and that the amount of salivation should be inversely related to the severity of halitosis. However, no previous studies have evaluated the relationship between hydration status and the severity of halitosis. Therefore, the purpose of the present study was to investigate how dehydration affects the severity of halitosis.

\section{MATERIALS AND METHODS}

\section{Participants}

A total of 20 healthy, young females were recruited for the study between December 1, 2014 and December 31, 2015. The participants with dental disease (gingival in$\operatorname{dex}>0$, plaque index $>0$ ), those currently taking medication, those who smoked, and those who displayed evidence of systemic illness were excluded.

\section{Ethical Considerations}

This study was reviewed and approved by the Institutional Review Board of Pusan National University Yangsan Hospital (IRB no. 05-2014-080). Written informed consent was obtained from each participant after providing complete information about the purpose and procedures of the study.

\section{Hydration and Dehydration of the Participants}

This study was conducted using a cross-over study method. Half of the subjects were induced to become dehydrated on the first day of the study and rehydrated on the following day. To induce relative dehydration, these subjects were instructed not to drink any fluids after dinner on the 
first day. On the second day, the subjects were limited to no more than $400 \mathrm{~mL}$ of water intake, including the water contained in food. After a one-day break for rehydration, all participants were instructed to overhydrate. Participants were instructed to drink $1 \mathrm{~L}$ of water on the evening of the third day. On the fourth day, participants were instructed to drink $3,400 \mathrm{~mL}$ of water to induce relative overhydration. On the afternoon of the second and fourth day, hydration status and severity of halitosis were assessed by an independent examiner. During the study period, participants were instructed to consume only foods and liquids from a provided list. The use of hair products containing perfume was prohibited on the days of evaluation, as was tooth brushing, mouth rinsing, eating, and drinking for 1 hour before assessment. The other half of the participants were hydrated on the first day of the study and dehydrated the following day. All participants were given the same type and amount of food on the day of evaluation.

The rationale for implementing a study with a crossover design was that this would provide a more efficient comparison of dehydrated, euhydrated, and overhydrated conditions. That is, fewer participants would be required with a crossover design because each patient would function as her own control.

\section{Measurement of Hydration Status}

Urine osmolality was measured to determine the level of hydration. Participants were graded as dehydrated, euhydrated, or overhydrated based on urine osmolalities of $\leq 500,501$ to 800 , and $\geq 801 \mathrm{m0sm} / \mathrm{kg}$, respectively [19].

\section{Measurement of Halitosis Severity}

All assessments were performed by a single physician not otherwise involved in this study.

\section{Organoleptic Assessment}

The severity of halitosis was determined by organoleptic assessment. This is a commonly used subjective method of measurement that involves the assessment of oral odor by others. Organoleptic scores were assigned using a previously described scale ( $0=$ no appreciable odor; $1=$ barely noticeable odor; $2=$ slight but clearly noticeable odor; $3=$ moderate odor; $4=$ strong odor; $5=$ foul odor) [20,21]. Organoleptic assessments were performed by a single physician independent of this study.

\section{Portable Gas Chromatography-Based Measurements}

To determine the concentrations of VSCs present within expiratory gas, a licensed dentist used a gas chromatograph (OralChroma). Exhaled gas samples were collected with a 1-mL plastic, disposable syringe that had been inserted into the oral cavity. The participants were instructed to close their mouths for 30 seconds before sample collection; 0.5 $\mathrm{mL}$ of expired air was then collected by the measuring device. After 8 minutes, the process was completed and the concentrations of the three gases were measured in units of $\mathrm{ng} / 10 \mathrm{~mL}$ or $\mathrm{nmol} / \mathrm{mol}$.

\section{BB Checker Measurements}

A thin-coated tin dioxide semiconductor gas sensor was used to detect reductive gases. This machine is sensitive to such gases as VSCs, hydrogen, ethanol, acetone, butylate, and ammonia. The BB Checker (Taiyo Instrument Inc., Osaka, Japan) expresses gas levels (BB values) based on olfactory intensity in humans according to the WeberFechner law. Olfactory intensity was quantified using a 0 to 100 scale. The sensor probe was covered with a disposable adaptor and was then inserted directly into the participants' mouths to prevent loss of sample gas. The adaptor prevented the sensor probe from directly contacting the oral mucosa. Before analysis, participants were instructed to keep their mouths closed and breathe through their noses only, for 180 seconds. The sensor was then placed in the center of the oral cavity. Oral air was measured for 15 seconds. For analysis of exhaled breath, participants were instructed to expel oral air, then inspire deeply, hold their breath for 15 seconds, and finally to exhale completely through the adapter over a 15 -second period.

\section{Assessment of Tongue Coating}

Tongue coating was assessed using a method previously described [21]. It was scored using a scale of 0 to 3, considering the thickness of the tongue plaque and the surface area covered ( $0=$ no visible coating; $1=$ thin coating on less than one-third of the back of the tongue; $2=$ thick coating on more than one-third of the back of the tongue; $3=$ thick coating). 


\section{Assessment of Salivation}

Whole saliva samples were collected over a 5-minute period in polypropylene tubes. Volumes of both resting or unstimulated saliva and stimulated saliva were measured in the afternoon of the second and fourth day, respectively. Saliva volumes were estimated by weighing the Salivette tubes (Sarstedt, Nümbrecht, Germany) immediately after collection and then subtracting the weight of the tube alone from this value. Weights were rounded to the nearest $\mathrm{mg}$ and a density of $1.00 \mathrm{~g} / \mathrm{mL}$ was assumed for the saliva [22]. Finally, saliva flow rate was calculated by dividing the saliva volume by the time required for collection.

\section{Statistical Analysis}

PASW (ver. 18.0 for Windows; SPSS Inc., Chicago, IL, USA) was used for statistical analysis. Statistical power analysis using GPower 3.1 (University of Düesseldorf, Düsseldorf, Germany) for sample size estimation was performed previously [23]. It was reported that 13 was the fewest number of participants required to detect a significant difference in the number of salivary antimicrobial proteins between individuals with exercise-induced dehydration with subsequent overnight fluid restriction and those without fluid restriction. The above study reported a modest correlation between dehydration and salivary mucosal immunity. Thus, our proposed sample size of 20 was more than adequate to meet the main study objective and allow for expected attrition.

The Shapiro-Wilk test was used to evaluate the normality of the distribution of the data. In this study, none of the measurements satisfied the normality condition. Therefore, we used the Kruskal-Wallis test to assess differences among more than two groups and the Mann-Whitney U test to assess differences between two groups. The correlation coefficient $\mathrm{R}$ was computed using Pearson's correlation coefficient analysis. p-values $<0.05$ were considered statistically significant.

\section{Participant Characteristics According to Hydration Status}

The demographic data for the 20 female participants are shown in Table 1 . The mean ages of participants in the dehydrated, euhydrated, and overhydrated groups were $29.30 \pm 3.30$, 33.00 \pm 6.06 , and $31.10 \pm 4.46$ years, respectively. The compositions of the three groups were similar with respect to age and body weight. One possible explanation for why some participants were classified as euhydrated was

Table 1. Participant characteristics according to hydration status ${ }^{\mathrm{a}}$

\begin{tabular}{|c|c|c|c|c|}
\hline Variable & Dehydration & Normal hydration & Over-hydration & p-value \\
\hline Number & 17 & 6 & 17 & - \\
\hline Age $(y)$ & $29.30 \pm 3.30$ & $33.00 \pm 6.06$ & $31.10 \pm 4.46$ & 0.883 \\
\hline Body weight $(\mathrm{kg})$ & $50.50 \pm 1.72$ & $51.50 \pm 2.38$ & $51.10 \pm 1.73$ & 0.867 \\
\hline Hydration status: urine osmolality (mOsm/kg) & $913.94 \pm 64.98$ & $749.50 \pm 40.00$ & $148.12 \pm 85.43$ & $<0.001$ \\
\hline
\end{tabular}

Values are presented as mean \pm standard deviation.

${ }^{\mathrm{a}}$ Kruskal - Wallis test.

Table 2. Halitosis severity according to hydration status

\begin{tabular}{|c|c|c|c|c|c|}
\hline \multicolumn{3}{|c|}{ Hydration status } & Dehydrated & Normal & Over-hydrated \\
\hline \multicolumn{3}{|c|}{ Halitosis severity* (organoleptic score) } & $1.50(1.25)^{\star \star \star}$ & $1.00(1.13)$ & $1.00(1.25)^{\star \star *}$ \\
\hline \multirow[t]{8}{*}{ Halitosis factor } & \multirow[t]{2}{*}{ Salivation rate $(\mathrm{mL} / \mathrm{min})$} & Resting & $0.30(0.46)$ & $0.25(0.50)$ & $0.50(0.57)$ \\
\hline & & Functional & $1.50(1.05)$ & $1.14(0.68)$ & $1.30(0.95)$ \\
\hline & \multirow{3}{*}{$\begin{array}{l}\text { Gas chromatography } \\
\text { (nmol/mol) }\end{array}$} & $\mathrm{H}_{2} \mathrm{~S}$ & $6.00(7.00)$ & $11.00(16.00)$ & $8.00(12.00)$ \\
\hline & & $\mathrm{CH}_{3} \mathrm{SH}$ & $0.00(0.00)$ & $3.50(38.25)$ & $0.00(3.00)$ \\
\hline & & $\left(\mathrm{CH}_{3}\right)_{2} \mathrm{~S}$ & $0.00(17.00)^{\star \star \star}$ & $3.00(18.75)$ & $0.00(4.50)^{* * *}$ \\
\hline & \multirow{3}{*}{$\begin{array}{l}\text { Tongue coating } \\
\text { BB checker (nmol/mol) }\end{array}$} & & $2.00(1.00)$ & $2.00(0.00)$ & $2.00(1.00)$ \\
\hline & & Oral gas & $20.00(26.50)$ & $11.50(8.75)$ & $14.00(19.50)$ \\
\hline & & Exhalation gas & $32.00(48.50)$ & $32.00(24.25)$ & $43.00(39.00)$ \\
\hline
\end{tabular}

Values are presented as median (interquartile range).

${ }^{*} p<0.05$, by Kruskal - Wallis test; ${ }^{* *} p<0.001$ by Mann - Whitney $U$ test. 
that these individuals did not follow the experimental protocol. The number of participants in each group ranged from 6 to 17 .

\section{RESULTS}

\section{Association between Dehydration and Halitosis}

The Kruskal-Wallis test revealed that hydration had a significant effect on organoleptic score (Table 2). That is, the scores in the overhydrated group were significantly lower than those in the other two groups. A significant difference in organoleptic score was found between the dehydrated and overhydrated groups (Mann-Whitney $U=41, Z=-3.636$, $\mathrm{p}<0.001, \mathrm{r}=-0.624$ with a Bonferroni correction). Although the difference between the dehydrated and euhydrated groups was insignificant, a large effect size was reported $(\mathrm{r}=-0.476)$. As shown in Fig. 1, our results demonstrated a moderate relationship between the degree of dehydration and organoleptic score $(\mathrm{r}=0.540, \mathrm{p}<0.001)$.

\section{Portable Gas Chromatography Analysis}

The values for $\mathrm{CH}_{3} \mathrm{SH}$ and $\left(\mathrm{CH}_{3}\right)_{2} \mathrm{~S}$ in the overhydrated
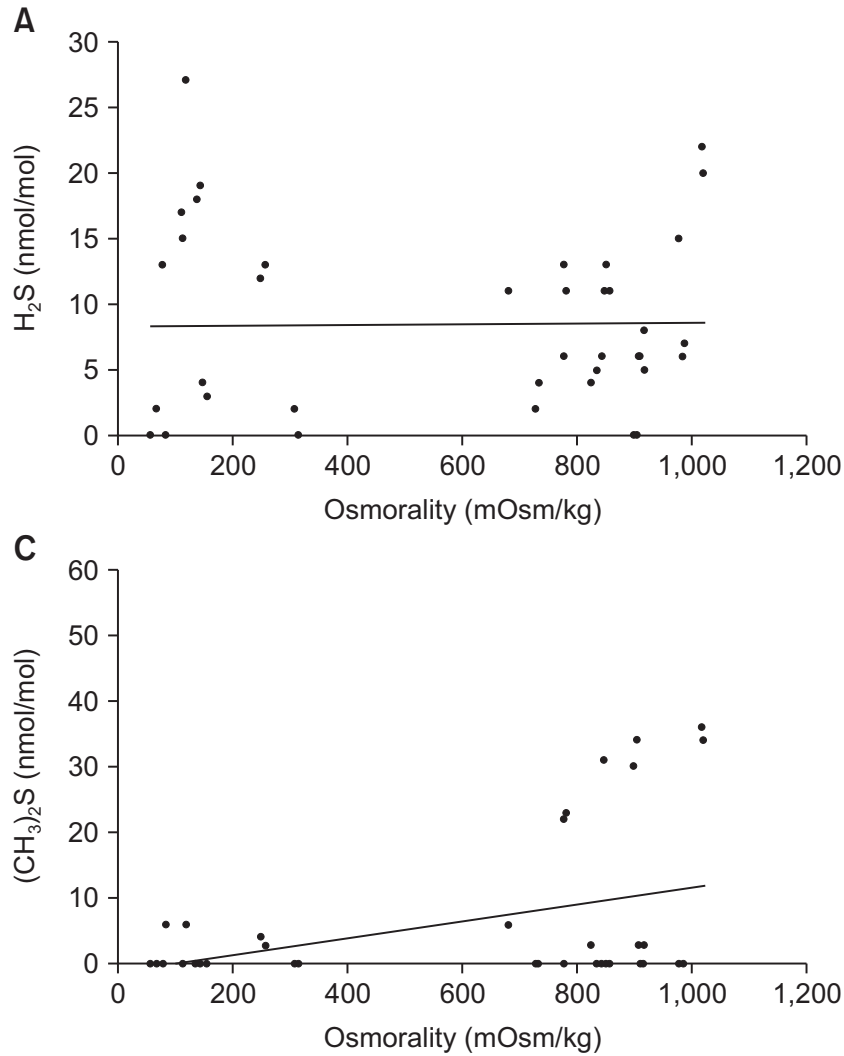

group were lower than those in the other two groups, but they were not significant (Table 2). However, $\left(\mathrm{CH}_{3}\right)_{2} \mathrm{~S}$ ( $r=0.410, p=0.009$ ) showed positive, but only moderate, correlations with the degree of dehydration (Fig. 2).

\section{Association between Dehydration, Salivation, and Tongue Coating}

Mean resting salivation rates in the dehydrated, euhydrated, and overhydrated groups didn't have a significant effect

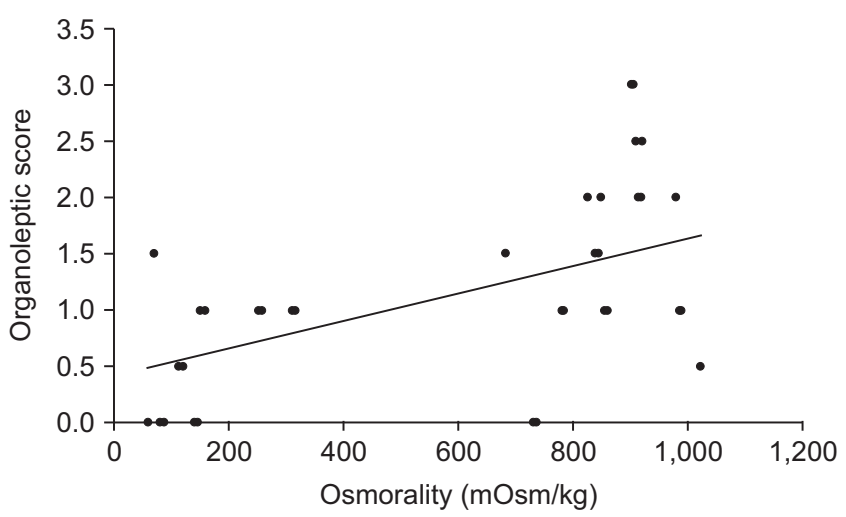

Fig. 1. Organoleptic scores according to the hydration status $(r=0.540, p<0.001 *)$. *Pearson's correlation coefficient.

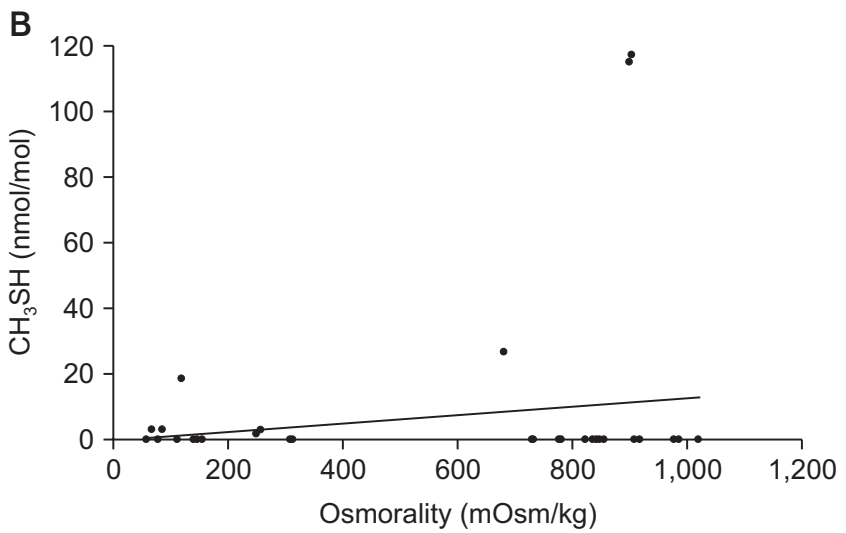

Fig. 2. The portable gas chromatography analysis of the participants according to the dehydration status. Concentrations of $\mathrm{H}_{2} \mathrm{~S}$ (A), $\mathrm{CH}_{3} \mathrm{SH}(\mathrm{B})$, and $\left(\mathrm{CH}_{3}\right)_{2} \mathrm{~S}(\mathrm{C})$ according to osmorality. $\left(\mathrm{CH}_{3}\right)_{2} \mathrm{~S}$ $\left(r=0.410, p=0.009^{*}\right)$ showed a positive correlation according to the dehydration status of the participants. *Pearson's correlation coefficient. 


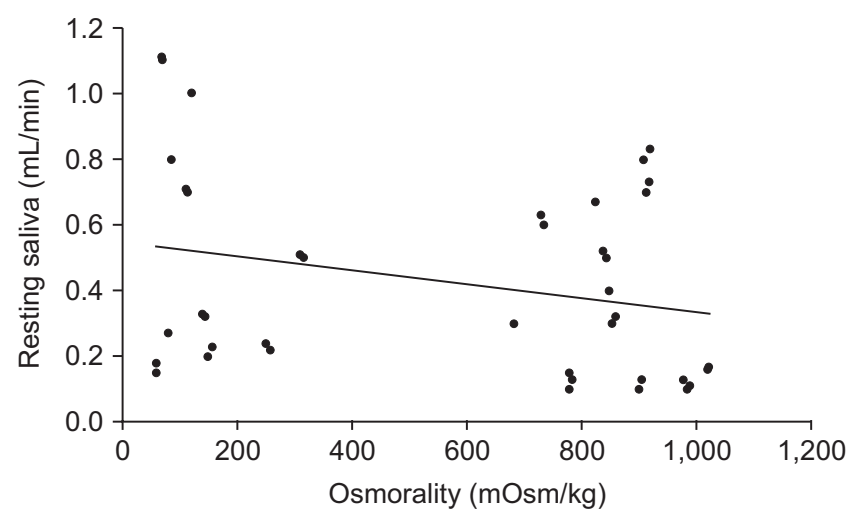

Fig. 3. Resting salivation measurements according to the dehydration status. There was a weak negative correlation between dehydration and resting salivation rate $(r=-0.267$, $p=0.096 *)$. *Pearson's correlation coefficient.

on mean stimulated salivation rates $(\mathrm{H}[2]=2.865, \mathrm{p}>0.05$; Table 2).

Resting and stimulated salivation rates in the overhydrated group were higher than those in the other two groups, and the amount of tongue coating was less. A negative correlation was found between the degree of dehydration and resting salivation rate $(r=-0.267, p=0.096$; Fig. 3), but no significant intergroup difference was found.

\section{DISCUSSION}

There is no single ideal test to objectively assess the severity of halitosis because there are many possible etiologies [24]. Any condition that favors the retention of anaerobic bacteria will predispose one to bad breath. Besides periodontal pockets, the most common site for the retention of bacteria is the dorsum of the tongue. Overnight and between meals, conditions in the oral cavity are optimal for odor production. In addition, systemic diseases, such as diabetes mellitus, uremia, and various hepatopathies, induce the formation of metabolic by-products that are detectable as halitosis. Usually, oral and oropharyngeal factors (tongue coating, gingivitis, periodontitis, and tonsillitis) and the bacterial formation of odorous VSCs, such as $\mathrm{H}_{2} \mathrm{~S}$ and $\mathrm{CH}_{3} \mathrm{SH}$, within the oral cavity are considered the major causes of halitosis [8]. In this sense, clearance of metabolic by-products by saliva appears to be an important factor in the reduction of halitosis. In our daily lives, dehydration is caused by insufficient water intake or by ingesting salty foods and caffeinated drinks. In these cases, relative dehydration occurs, which might be a cause of halitosis. To date, no guideline has been issued with respect to water intake for the reduction of halitosis.

We studied the relationship between hydration status and halitosis by inducing relative dehydration. In this study, all participants were young and healthy female volunteers without any evidence of oral or systemic illness. In such subjects, even if relative dehydration is achieved, systemic compensation may occur, and thus, assessment of the severity of dehydration might not be sufficient to evaluate the relationship between hydration and halitosis. Nevertheless, we explored the idea that if the participants were dehydrated, then resting salivation would be decreased, and thus the volume of saliva would be reduced.

We observed that the degree of dehydration was positively correlated with the severity of halitosis. In fact, when the participants were dehydrated, noticeable halitosis was observed in most of them (an organoleptic score of more than 1). Under such circumstances, water is believed to be redistributed to more central organs, such as the kidneys and urinary tract, and thus, salivation is decreased. The relative decrease in salivation might be one of the factors causing halitosis due to the decreased clearance of metabolic byproducts [25].

In this study, the mean values for salivation in the dehydrated group were not significantly lower than those in the euhydrated or overhydrated groups. This might be due to the redistribution of water possible in a young, healthy person [26].

The fact that the amount of tongue coating in the dehydrated group was greater than that of the other groups implies that the function of the saliva was decreased even if the amount was not decreased significantly [27]. This means that relative dehydration could cause halitosis even in young, healthy females.

VSC formation by bacteria in the oral cavity or oropharynx can be objectively measured by portable gas chromatography, and currently, VSCs are considered an important causative factor for halitosis. These sulfur compounds could also be manifestations of systemic disease, such as hepatic failure, or isolated persistent hypermethioninemia [7,28-31]. Treatment with cysteamine [24,32-34] or dimethyl sulfoxide medications [12] has also been implicated in the formation 
of VSCs. In the present study, although the correlation between VSCs and dehydration was not strong, $\left(\mathrm{CH}_{3}\right)_{2} \mathrm{~S}$ was found to be positively correlated with the degree of dehydration ( $\mathrm{r}=0.410$ in Table 2 and Fig. 2). Tangerman and Winkel32 reported that the VSC, $\mathrm{CH}_{3} \mathrm{SH}$ and $\mathrm{H}_{2} \mathrm{~S}$ are the main contributors to intra-oral halitosis [35]. On the other hand, $\left(\mathrm{CH}_{3}\right)_{2} \mathrm{~S}$ is the main contributor to extra-oral or bloodborne halitosis and not attributed to recent ingestion of volatile foodstuffs [36]. Judging by the fact that $\left(\mathrm{CH}_{3}\right)_{2} \mathrm{~S}$ is a factor in blood-borne halitosis, $\left(\mathrm{CH}_{3}\right)_{2} \mathrm{~S}$ can be diluted with water-related hemodilution, which may explain our results.

In this study, the degree of dehydration appeared to be positively correlated with the severity of halitosis and the formation of VSCs $\left(\left[\mathrm{CH}_{3}\right]_{2} \mathrm{~S}\right)$ which are mediated by salivary action directly and indirectly. According to our results, water intake appears to be a crucial factor in causing halitosis, even in young female participants. Thus it is appropriate to encourage adequate water intake to remain hydrated and to reduce some of the physiologic causes of halitosis [25,37].

This study had several limitations. First, we attempted to control for causes of halitosis other than water intake, but unpredictable factors might be involved. Second, since this study enrolled only 20 young female volunteers, the sample size may not be sufficient to allow a generalization of our findings. Third, as an objective clinical indicator for dehydration status, serum osmolality is currently mainly used as a diagnostic criterion, whereas urine osmolality was used in our study due to the advantage of a non-invasive test. However, it cannot be regarded as a very high-accuracy test method, so caution is required in interpreting the results [38].

Based on the results of this study, we suggest that further studies be conducted to study the mechanism of physiological halitosis reduction according to hydration status through analysis of changes in protein, immunoglobulin or enzymes in saliva as water intake is increased. Additionally, it would be helpful to increase the number of participants, including males and participants of various ages.

In this study, hydration status was moderately correlated with halitosis and VSC formation in young, healthy females. The degree of dehydration could be a contributing factor to physiological halitosis. Dehydration should be considered a possible etiology of halitosis.

\section{CONFLICT OF INTEREST}

No potential conflict of interest relevant to this article was reported.

\section{ORCID}

\author{
Soo-Min Ok \\ https://orcid.org/0000-0003-1776-371X \\ Sung-Hee Jeong \\ https://orcid.org/0000-0002-6296-4775 \\ Chang-Hyung Lee \\ https://orcid.org/0000-0001-6460-6368
}

\section{REFERENCES}

1. Scully C, Greenman J. Halitology (breath odour: aetiopathogenesis and management). Oral Dis 2012;18:333-345.

2. Scully C. Halitosis. BMJ Clin Evid 2014;2014:1305.

3. Aydin M, Harvey-Woodworth CN. Halitosis: a new definition and classification. Br Dent J 2014;217:E1.

4. Ashwath B, Vijayalakshmi R, Malini S. Self-perceived halitosis and oral hygiene habits among undergraduate dental students. J Indian Soc Periodontol 2014;18:357-360.

5. Ostberg AL, Halling A, Lindblad U. Gender differences in knowledge, attitude, behavior and perceived oral health among adolescents. Acta Odontol Scand 1999;57:231-236.

6. Al-Omiri MK, Barghout NH, Shaweesh AI, Malkawi Z. Level of education and gender-specific self-reported oral health behavior among dental students. Oral Health Prev Dent 2012;10:29-35.

7. Tangerman A. Halitosis in medicine: a review. Int Dent J 2002;52 Suppl 3:201-206.

8. Akaji EA, Folaranmi N, Ashiwaju 0. Halitosis: a review of the literature on its prevalence, impact and control. Oral Health Prev Dent 2014;12:297-304.

9. Tangerman A, Winkel EG. Intra- and extra-oral halitosis: finding of a new form of extra-oral blood-borne halitosis caused by dimethyl sulphide. J Clin Periodontol 2007;34:748-755.

10. Yaegaki K, Coil JM. Examination, classification, and treatment of halitosis; clinical perspectives. J Can Dent Assoc 2000;66:257261.

11. Salako NO, Philip L. Comparison of the use of the Halimeter and the Oral Chroma $^{\mathrm{TM}}$ in the assessment of the ability of common cultivable oral anaerobic bacteria to produce malodorous volatile sulfur compounds from cysteine and methionine. Med Princ Pract 2011;20:75-79.

12. Tangerman A, Winkel EG. Extra-oral halitosis: an overview. J Breath Res 2010;4:017003.

13. Dawes C, Pedersen AM, Villa A, Ekström J, Proctor GB, Vissink A, et al. The functions of human saliva: a review sponsored 
by the World Workshop on Oral Medicine VI. Arch Oral Biol 2015;60:863-874.

14. Dawes C. Salivary flow patterns and the health of hard and soft oral tissues. J Am Dent Assoc 2008;139 Suppl:18S-24S.

15. Chaudhury NM, Shirlaw P, Pramanik R, Carpenter GH, Proctor GB. Changes in saliva rheological properties and mucin glycosylation in dry mouth. J Dent Res 2015;94:1660-1667.

16. Saleh J, Figueiredo MA, Cherubini K, Salum FG. Salivary hypofunction: an update on aetiology, diagnosis and therapeutics. Arch Oral Biol 2015;60:242-255.

17. Walsh NP, Montague JC, Callow N, Rowlands AV. Saliva flow rate, total protein concentration and osmolality as potential markers of whole body hydration status during progressive acute dehydration in humans. Arch Oral Biol 2004;49:149-154.

18. Tenovuo J. Clinical applications of antimicrobial host proteins lactoperoxidase, lysozyme and lactoferrin in xerostomia: efficacy and safety. Oral Dis 2002;8:23-29.

19. Perrier ET, Buendia-Jimenez I, Vecchio M, Armstrong LE, Tack I, Klein A. Twenty-four-hour urine osmolality as a physiological index of adequate water intake. Dis Markers 2015;2015:231063.

20. Schemel-Suárez M, Chimenos-Küstner E, Estrugo-Devesa A, Jané-Salas E, López-López J. Halitosis assessment and changes in volatile sulfur compounds after chewing gum: a study performed on dentistry students. J Evid Based Dent Pract 2017;17:381-388.

21. Tanabe-Ikegawa M, Takahashi T, Churei H, Mitsuyama A, Ueno T. Interactive effect of rehydration with diluted sports drink and water gargling on salivary flow, $\mathrm{pH}$, and buffering capacity during ergometer exercise in young adult volunteers. J Oral Sci 2018;60:269-277.

22. da Silva Modesto KB, de Godói Simões JB, de Souza AF, Damaceno N, Duarte DA, Leite MF, et al. Salivary flow rate and biochemical composition analysis in stimulated whole saliva of children with cystic fibrosis. Arch Oral Biol 2015;60:1650-1654.

23. Fortes MB, Diment BC, Di Felice U, Walsh NP. Dehydration decreases saliva antimicrobial proteins important for mucosal immunity. Appl Physiol Nutr Metab 2012;37:850-859.

24. Lee SS, Zhang W, Li Y. Halitosis update: a review of causes, diagnoses, and treatments. J Calif Dent Assoc 2007;35:258-260, 262, 264-268.

25. Ship JA, Fischer DJ. The relationship between dehydration and parotid salivary gland function in young and older healthy adults. J Gerontol A Biol Sci Med Sci 1997;52:M310-M319.
26. Mack GW, Weseman CA, Langhans GW, Scherzer H, Gillen CM, Nadel ER. Body fluid balance in dehydrated healthy older men: thirst and renal osmoregulation. J Appl Physiol (1985) 1994;76:1615-1623.

27. Gross CR, Lindquist RD, Woolley AC, Granieri R, Allard K, Webster B. Clinical indicators of dehydration severity in elderly patients. J Emerg Med 1992;10:267-274.

28. Tangerman A. Measurement and biological significance of the volatile sulfur compounds hydrogen sulfide, methanethiol and dimethyl sulfide in various biological matrices. J Chromatogr B Analyt Technol Biomed Life Sci 2009;877:3366-3377.

29. Singh VP, Malhotra N, Apratim A, Verma M. Assessment and management of halitosis. Dent Update 2015;42:346-348, 351353.

30. van den Broek AM, Feenstra L, de Baat C. A review of the current literature on aetiology and measurement methods of halitosis. J Dent 2007;35:627-635.

31. Van den Velde S, Nevens F, Van Hee P, van Steenberghe D, Quirynen M. GC-MS analysis of breath odor compounds in liver patients. J Chromatogr B Analyt Technol Biomed Life Sci 2008;875:344-348.

32. Besouw M, Blom H, Tangerman A, de Graaf-Hess A, Levtchenko E. The origin of halitosis in cystinotic patients due to cysteamine treatment. Mol Genet Metab 2007;91:228-233.

33. Torsten M, Gómez-Moreno G, Aguilar-Salvatierra A. Drug-related oral malodour (halitosis): a literature review. Eur Rev Med Pharmacol Sci 2017;21:4930-4934.

34. Bollen CM, Beikler T. Halitosis: the multidisciplinary approach. Int J Oral Sci 2012;4:55-63.

35. Lee CH, Kho HS, Chung SC, Lee SW, Kim YK. The relationship between volatile sulfur compounds and major halitosis-inducing factors. J Periodontol 2003;74:32-37.

36. Harvey-Woodworth CN. Dimethylsulphidemia: the significance of dimethyl sulphide in extra-oral, blood borne halitosis. Br Dent J 2013;214:E20

37. Kim Y, Ma D. Descriptive statistical analysis report on the results of questionnaire and observations on patients with physiological halitosis who visited the KUMC halitosis clinic- a retrospective study. Research Square. 1066177 [Preprint]. 2021 [cited 2021 Nov 12]. Available from: https://doi.org/10.21203/rs.3.rs-1066177/v1.

38. Armstrong LE. Assessing hydration status: the elusive gold standard. J Am Coll Nutr 2007;26(5 Suppl):575S-584S. 